\title{
Gradhiva
}

GRADHIV

Revue d'anthropologie et d'histoire des arts

22 | 2015

Cosmos

\section{Choses cosmiques et cosmogrammes de la technique}

Cosmograms and Other Cosmic Things

John Tresch

Traducteur : Josiane Massard-Vincent

\section{OpenEdition}

\section{Journals}

Édition électronique

URL : http://journals.openedition.org/gradhiva/3019

DOI : 10.4000/gradhiva.3019

ISSN : $1760-849 x$

Éditeur

Musée du quai Branly Jacques Chirac

Édition imprimée

Date de publication : 1 octobre 2015

Pagination : $24-47$

ISBN : 978-2-35744-092-0

ISSN : 0764-8928

\section{Référence électronique}

John Tresch, « Choses cosmiques et cosmogrammes de la technique », Gradhiva [En ligne], 22 | 2015, mis en ligne le 01 octobre 2018, consulté le 04 mai 2019. URL : http://journals.openedition.org/ gradhiva/3019; DOl : 10.4000/gradhiva.3019 


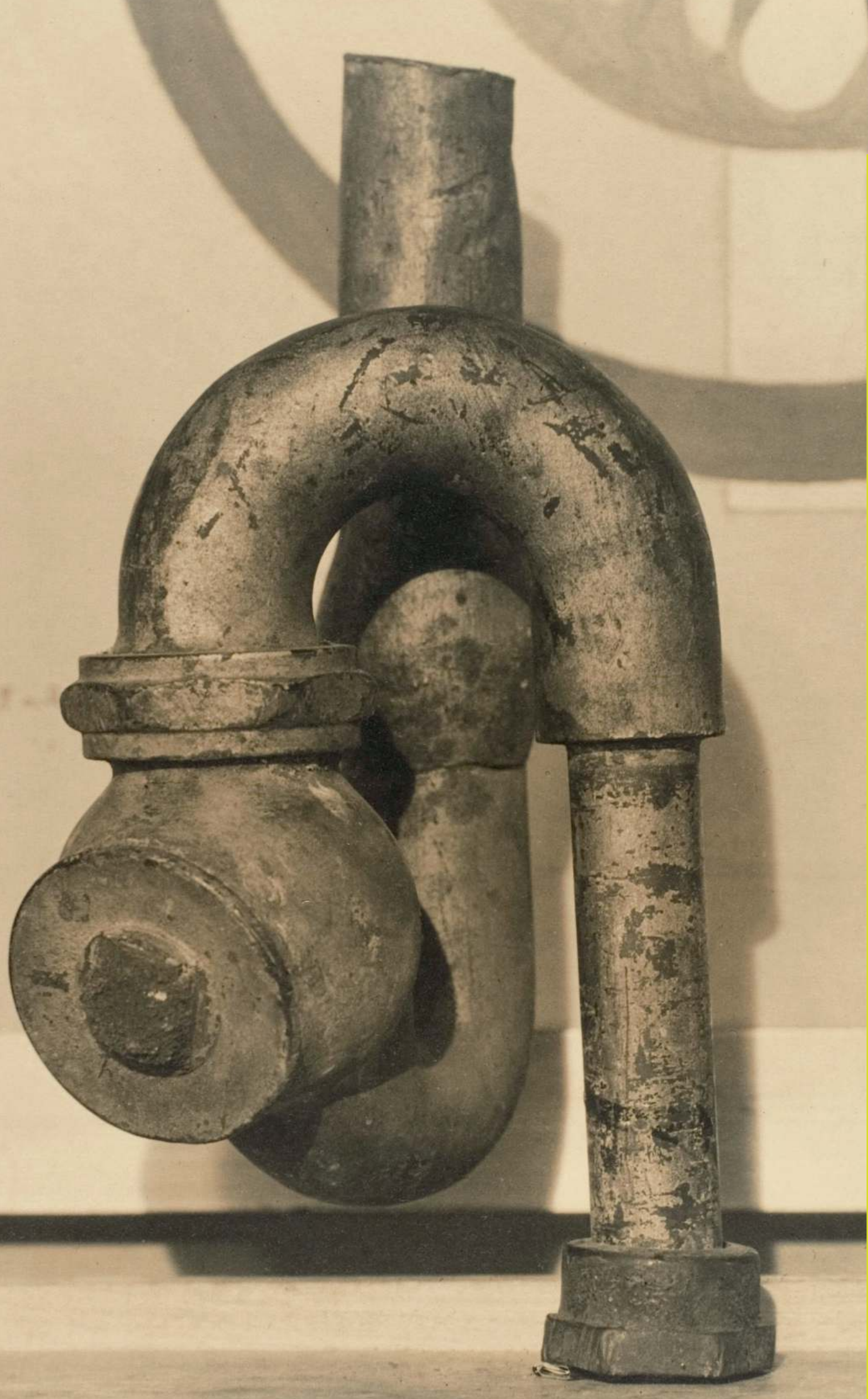

홍 


\section{Choses cosmiques et cosmogrammes de la technique *}

par John Tresch

Les choses peuvent évoquer et même contenir tout un cosmos: c'est la thèse de Martin Heidegger dans certains de ses écrits tardifs, qui parlent des choses en tant que « rassemblements ». Mais son analyse des choses s'inscrit dans une critique soutenue de la technologie et de l'«époque des conceptions du monde ".

Dans cet article, je considère les objets techniques, eux aussi, comme des «choses cosmiques » à la Heidegger. Pour contrebalancer ses généralisations totalisantes, voire totalitaires, sur l'« arraisonnement ", ainsi qu'une tendance, assez largement répandue parmi les critiques de la modernité, à ne présenter la technologie qu'en termes dystopiques, uniformes et claustrophobes, je propose le concept de «cosmogramme»: un objet concret qui cherche à résumer l'ordre du cosmos. Les cosmogrammes des sciences et de la technique occidentales peuvent servir de base à la comparaison et à la connexion du «monde industriel » avec d'autres manières d'organiser l'univers. 
1. Voir Hughes 2004.

Parmi les nombreuses exceptions aux études de la technologie où priment les "rouages" dans un univers défini comme un "mécanisme d'horlogerie". voir Marx 2000 ; Noble

1999; les chapitres sur «la cosmologie et la technologie in Cardwell 1972,

en particulier p. 127-163. Voir aussi les travaux à inflexion anthropologique

sur la science

et la technologie,

notamment ceux de

Hugh Gusterson,

Donna Haraway, Stefan

Helmreich, Bruno Latour, Paul Rabinow, Sharon

Traweek et leurs adeptes.

\section{Un monde dans une vitrine}

En parcourant un musée d'anthropologie, avec ses diaporamas et ses vitrines remplies d'outils, de masques, de statues et d'armes qui condensent des formes entières de vie, ne vous êtes-vous jamais demandé à quoi ressemblerait une vitrine consacrée à la culture contemporaine de l'Amérique du Nord ou des États-Unis? Elle inclurait forcément des symboles et objets évoquant une pluralité contestée de systèmes religieux, politiques et scientifiques. Une place éminente devrait aussi y être faite aux objets techniques: ordinateurs, téléphones portables, pilules, modèles réduits d'usines, avions et autoroutes, avec, qui sait, l'ombre menaçante de bombes nucléaires dans un ciel peint en bleu. Le plastique mat et le titane étincelant de ces objets trancheraient avec le bois, les coquillages, les plumes et l'écorce des vitrines voisines; pourtant, ce sont ces choses - à l'instar des calendriers maya, statues songye nkisi, canoés et totems kwakiutl qui définissent notre cosmologie, nos rapports entre hommes et à la nature.

Une cosmologie est plus qu'un système de classification, un mythe d'origine ou une théorie des relations entre les composantes de l'univers; elle implique aussi des dimensions affectives, esthétiques, et la perception d'une cohérence entre les mots, usages et objets spécifiques à un groupe (Viveiros de Castro 2012). Souvent, cependant, l'image que l'on nous sert de la «cosmologie», ou "vision du monde», rattachée à la technologie moderne est beaucoup plus mince que celle offerte par l'anthropologie une «mécanisation de l'image du monde» réduisant toute expérience à un jeu de parpaings, c'est-à-dire à un rapport de masses et de forces, de moyens et de fins fonctionnalistes (Dijksterhuis 1961). La crainte que la réduction et l'instrumentalisation «positivistes" n'en viennent à dominer la totalité de la vie humaine parcourt les écrits critiques sur la technologie. Dans cette tradition, notamment en ce qu'elle a pris en compte la destruction causée par les guerres du $x x^{e}$ siècle, les machines sont souvent assimilées à la quête abstraite et brutale d'efficacité et d'exploitation $\mathbf{1}$.

Les derniers travaux de Martin Heidegger ont poussé cette tendance à l'extrême. Même le langage néokantien d'«image du monde » ou de «vision du monde», soutient-il, porte en lui l'agression qu'implique la relation technologique à la nature: «Le processus fondamental des temps modernes, c'est la conquête du monde en tant qu'image conçue. » (Heidegger 1962b: 123) Avec l'apparition d'un «sujet» à qui toute la nature doit être représentée, l'homme, «seigneur de la terre», a la voie libre pour donner l'«assaut». «Dans l'impérialisme planétaire de l'homme organisé technologiquement, le subjectivisme de l'homme atteint son point culminant, à partir duquel il entrera dans le nivellement de l'uniformité organisée pour s'y installer à demeure; car cette uniformité est l'instrument le plus sûr de l'empire complet, parce que technique, sur la terre. " (Ibid.: 123-144) Les œuvres tardives de Heidegger brossent à grands traits cette image terrifiante de toute la technologie et de la science moderne. Selon de nombreux auteurs, sa déception envers le régime nazi, qu'il avait notoirement soutenu dans les années 1930, se serait muée après la Seconde Guerre mondiale en une aversion pour la société industrielle dans son ensemble. Les lecteurs de Heidegger ont eu un impact indirect sur les études consacrées à la science et la technologie - en premier lieu, peut-être, à travers les écrits de Michel 
Foucault qui a exploité nombre de ses idées fondamentales au profit de finalités inattendues; longtemps au cœur de la philosophie critique de la technologie, les idées de Heidegger sur les choses ont connu récemment un regain d'intérêt, toutefois mineur et circonspect ${ }^{2}$. Bien que je ne sois ni un spécialiste ni un dévot de son œuvre, le thème de ce volume me paraît propice à un réexamen de ses arguments concernant la technologie et, surtout, les «choses».

Si la philosophie de Heidegger peut intéresser les ethnologues, c'est pour une autre raison. Née de la même tradition postkantienne qui a engendré l'anthropologie culturelle, elle offre une réponse aux critiques du concept anthropologique de "culture». En effet, le monde qu'elle présente n'est pas un système, avant tout clos et idéalisé, de symboles, de théories ou de croyances, mais plutôt un tissu interconnecté d'objets et d'usages qui sont publics et partagés sans pouvoir être pleinement articulés ou rationalisés. Cet aspect de son travail a été rapproché de la "forme de vie" du Wittgenstein tardif et de ses arguments contre la "théorie de l'image" rapportée au langage. Sans même parler des échos existentiels d'Être et Temps, l'ensemble des travaux de Heidegger abordent la dimension imaginative, affective et holistique des objets que la plupart des disciples de Wittgenstein passent sous silence ${ }^{3}$.

Comme l'indique la discussion de Heidegger sur l'«époque des conceptions du monde ", ce holisme a cependant induit parfois des généralités totalisantes - voire totalitaires. Afin de corriger la tendance à ne présenter la technologie moderne qu'en termes dystopiques, uniformes et claustrophobes, cet essai examinera deux types d'objets techniques: les choses cosmiques et les cosmogrammes. Le premier envisage comment un objet ordinaire peut contenir un cosmos entier, le second, comment un cosmos peut être traité tout simplement comme une chose. Ensemble, ils ouvrent la voie à des réflexions sur le cosmos créé par les objets techniques. Dans l'esprit d'un «essai ", les notions de chose cosmique et de cosmogramme autorisent la comparaison et la connexion entre le «monde industriel» et d'autres modes d'organisation de l'univers.

\section{Équipement, monde, choses}

Les travaux de Heidegger sont denses, de grande envergure et, par bien des aspects, rébarbatifs. La discussion qui suit, restreinte à une poignée de concepts dans son œuvre - équipement, monde, œuvres d'art et choses -, est simplement centrée sur certaines de ses idées, autant que sur ses omissions, au sujet du rapport entre objets technologiques et cosmologie.

La première moitié d'Être et Temps est focalisée sur les ententes tacites mises en œuvre dans l'activité ordinaire et les tissus de significations ni verbalisés ni analysés qui précèdent la connaissance "théorique" ou "objective"; ces arguments anticipent de multiples façons les perspectives actuelles des études sur la science et la technologie ${ }^{4}$. Plus fondamentalement, son "analyse existentiale du Dasein" a rejeté l'hypothèse traditionnelle de la philosophie selon laquelle la relation élémentaire au monde est celle d'un sujet qui se «représente» des objets dans
2. Le style résolument ardu de Martin Heidegger et la tache indélébile de son soutien au nazisme, autant que son supposé mauvais caractère, rendent ambivalente toute référence à ses travaux; voir Farias 1987 ; Gordon 2010. Pourtant, pour ceux qui ont trouvé des idées utiles dans Michel Foucault,

Giorgio Agamben, Hannah Arendt, Jacques Derrida, Pierre Bourdieu et bien d'autres, une confrontation directe avec cette source paraît inévitable.

Sur son importance pour la philosophie de la technologie et l'épistémologie, voir lhde 2004: 117-131; Rouse 1987 ; Daston 2004 : 16 ;

Latour et Weibel (éd.) 2005 ; Harman 2010 (et la vague d'études des "objets" que ce dernier inspire).

L'héritage de Heidegger se retrouve aussi dans la théorie des média (Kittler 1992), la «sphérologie» et les études de

l'«anthropotechnique» de Peter Sloterdijk.

Les travaux de Heidegger sont aussi devenus une référence dans les études postcoloniales; voir Mitchell 1988; Chakrabarty 2000
3. À l'exception de Stanley Cavell, qui en tant que néophyte en philosophie analytique pourrait bien "confirmer la règle".

Sur Wittgenstein vs Heidegger, voir Rorty 1993. Dans cette optique, l'anthropologie de Clifford Geertz peut apparaître comme une tentative pour concilier le positivisme et la phénoménologie: dans un texte au titre emprunté au philosophe wittgensteinien Gilbert Ryle, Geertz décrit la fascination de l'anthropologie pour l'exotisme en langage heideggerien comme " un expédient visant à échapper à la monotonie d'un monde familier qui nous dissimule le mystère de notre propre capacité à entrer en relation manifeste les uns avec les autres " (Geertz 1998: IV, 23).

4. Voir les remarques de Heidegger sur «l'interprétation existentiale de la science ", Heidegger 1986 : 347. 


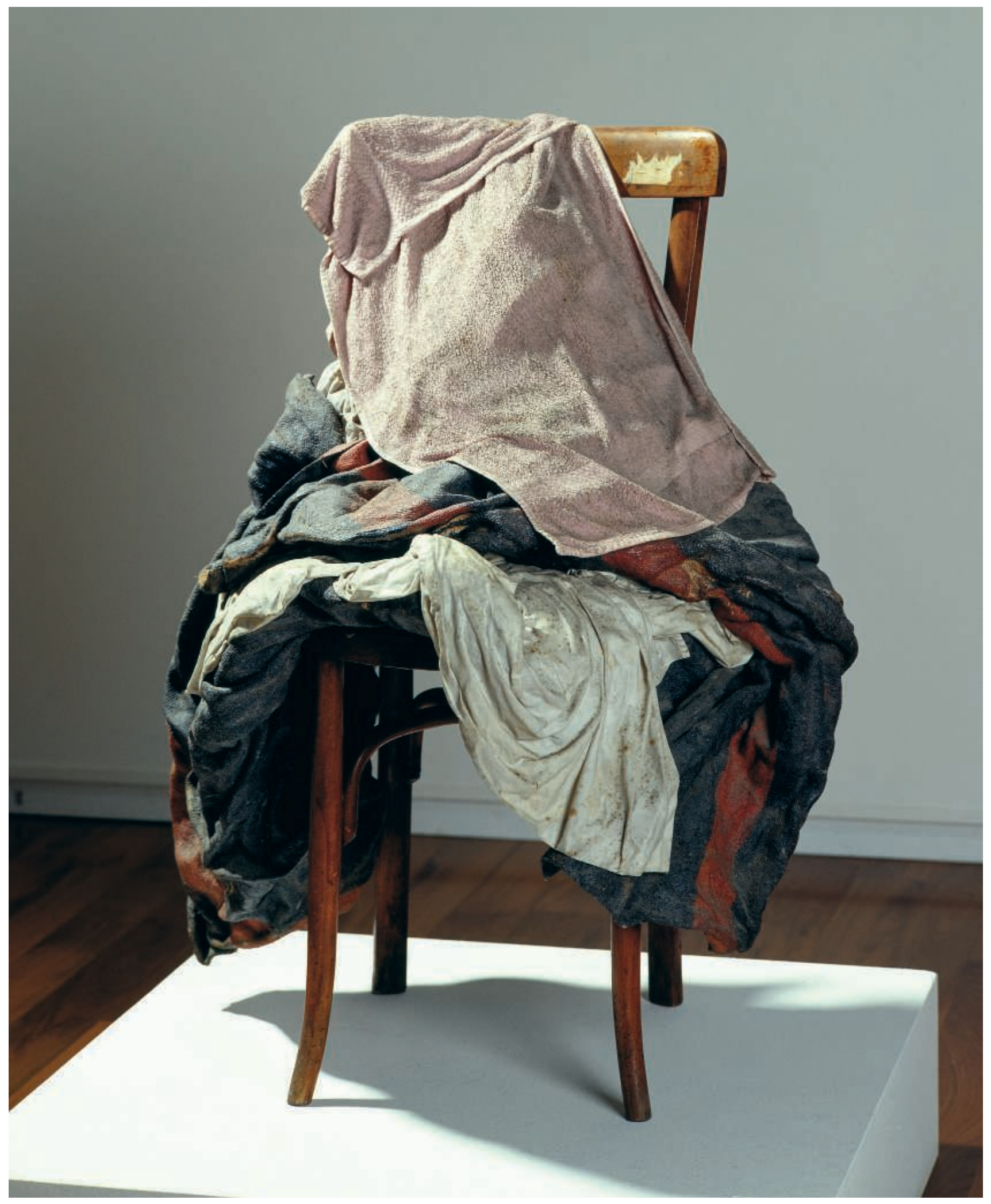


des concepts, des propositions ou des croyances. Au lieu de commencer par un sujet percevant, synthétisant ou interprétant, Heidegger a débuté avec le Dasein, l'«être-là : une immersion dans des pratiques et des interactions habituelles qui sont «comprises» sans avoir besoin de formulation explicite, de réflexion délibérée ou d'interprétation consciente. Ces pratiques sont le soubassement de notre thématisation explicite de tout objet ou, d'ailleurs, de tout «sujet». «Être-au-monde» - ce soubassement tacite de l'équipement, des références, lieux et visées - est le moyen par lequel l'être est dévoilé dans le Dasein.

Ce soubassement est d'ordinaire «transparent» dans nos usages. Comme dans la célèbre discussion d'Être et Temps sur l'équipement (Zeug), le marteau qu'on prend est simplement un moyen disponible, à portée de main (zuhanden), en vue d'une fin, par exemple accrocher un tableau. Mais quand le marteau est indisponible, cassé, ou trop lourd pour la tâche, ses caractéristiques propres sont soudain, de manière détestable, manifestes: l'outil devient vorhanden, c'est-à-dire «advenant», «étant sous la main» ou « objectivement présent 5 » (Heidegger 1986: 62-67). De telles pannes permettent l'«attitude théorique» qui envisage les objets hors de leurs contextes et usages ordinaires; c'est le point de départ du savoir scientifique.

En même temps, l'équipement cassé nous fait prendre conscience du système de références dont il fait partie: le «monde», ou le contexte de significations et de buts qui est «là» avant que quiconque l'ait observé ou vérifié, devient manifeste. Dans ses œuvres tardives, Heidegger affirme que la «clairière insaisissable» qui ouvre un monde est différente pour chaque époque ${ }^{6}$ (ibid.: 170). L'Origine de l'œuvre d'art prend acte de ce passage à I'historicité avec une discussion sur le tableau de Vincent van Gogh représentant une vieille paire de chaussures et la manière dont il dévoile le monde du paysan: «Une paire de souliers de paysan, et rien de plus. Et pourtant... Dans l'obscure intimité du creux de la chaussure est inscrite la fatigue des pas du labeur. Dans la rude et solide pesanteur du soulier est affermie la lente et opiniâtre foulée à travers champs, le long des sillons toujours semblables, s'étendant au loin sous la bise. » (Heidegger 1962a: 34) Le cadre du tableau fonctionne d'une manière analogue à la panne d'outillage. Il délimite un espace dans une "pratique focale» qui nous permet de voir le mode d'être spécifique d'une entité individuelle. Dans le cas des chaussures, «c'est bien plus l'être-produit du produit qui arrive seulement par l'œuvre et seulement dans l'œuvre, à son paraître » (ibid. : 36). De plus, tout comme la panne d'équipement rend visible l'ensemble de références de l'outil, l'œuvre d'art dévoile la clairière historiquement située dans laquelle les entités sont révélées pour une époque: dans un autre exemple, un temple grec «ouvre un monde et en maintient à demeure le règne ${ }^{7}$ » (ibid. : 47).

Bizarrement, pourtant, ce glissement vers la spécificité historique a conduit Heidegger à certaines de ses généralisations les plus grossières ${ }^{8}$. Selon ses travaux tardifs, notre époque - en ce qu'elle s'oppose à la Grèce, à Rome ou au Moyen Âge - est définie par la technologie. Sa manière spécifique de révéler l'être est l'arraisonnement (Gestell) qui nous pousse vers le monde de telle façon que tout nous apparaît simplement comme un «fonds" - un stock de ressources à mettre en ordre et en usage. L'arraisonnement,
5. Les expressions entre guillemets sont des versions des traductions françaises de vorhanden. La notion de "transparence» dans les études de la science et de la technologie trouve ici une anticipation (Schaffer 1989: 70). Graham Harman (2002) souligne la répétition par Heidegger de la distinction zuhanden/ vorhanden.

6. Gilles Deleuze remarque les nuances heideggeriennes des épistèmes ou "a priori historiques " de Foucault, mais voit aussi une différence significative dans son insistance sur l'écart entre le visible et le dicible - un "être-langage" de régularités discursives et un «être-lumière » de formes architecturales (Deleuze 1986: 55-75, 115-130).

\section{Remarque}

terminologique: dans cet essai, je n'ai pas cherché à éviter l'ambiguïté entre les termes «monde» et "cosmos" que Heidegger lui-même distingue: "cosmos" ou «nature», comme totalité des êtres, est moins essentiel que "être-au-monde» (1986: 66 ; 1962b: 116)

\section{ci-contre}

fig = 1

Antoni Tàpies, Cadira roba, 1970, assemblage, $94 \times 76 \times 63 \mathrm{~cm}$. Fundaciò Antoni Tàpies, Barcelone, inv. T-1481. Photo VEGAP (c) ADAGP, Paris 2015.
Pourtant, son concept de «monde" est à peine moins cosmique que "cosmos": dans Être et Temps, il spécifie plusieurs sens pour «monde» (1986: 61) et prétend se concentrer sur «monde» en tant que région délimitée d'étants. Hubert Dreyfus, cependant, montre que son usage glisse de "monde» à "contexte local » ou région unique d'étants à une "unique totalité englobante" (Dreyfus 1992: 181). Les questions terminologiques sont étroitement liées à des questions architectoniques: on peut noter aussi bien une distinction verticale (ou "différence ontologique») entre des étants considérés du point de vue ontique et l'"être " que des distinctions horizontales entre des régions ontiques au sein d'un même «monde" englobant (différentes sciences, domaines culturels, ou peut-être les "formations discursives " de Foucault) ou entre plusieurs de ces «mondes» (ce qu'on pourrait appeler des relations interculturelles).

8. Jürgen Habermas nomme cette tendance "abstraction via essentialisation " (1992: 198). 
9. Sur la compréhension des objets externes, voir Heidegger 1958c.

10. Selon l'une des bêtes noires néokantiennes de Heidegger, Wilhlem Dilthey, un Weltbild, ou image du monde partie de l'expérience vécue, peut, après réflexion, être élaboré en une conception systématisée du monde ou Weltanschauung; dans les deux cas, cela reste une représentation interne. Pour Heidegger, la notion d'une vision du monde grecque ou médiévale est contradictoire puisque, en ces temps-là, " sujet » et "objet » n'avaient pas encore été convoqués comme les principaux acteurs du drame moderne entre la conscience humaine et le monde extérieur.

11. Voir aussi Heidegger 1958b: 176-177. Pour un débat apparenté sur les «interprétations cosmologiques" et les "effets d'épiphanie " dans les choses quotidiennes, voir Gumbrecht 2000. Dans Making Things Public (2005), Bruno Latour et Peter Weibel, avec un intérêt implicite pour ce qu'lsabelle Stengers appelle la "cosmopolitique", empruntent le parcours étymologique de Heidegger sur la "chose" comme "rassemblement » le long de chaînes de dérivation et rattachent res (en latin, "chose", et racine de "réalité ") à la "chose publique", res publica ou «république», d'où le jeu de mots de leur titre. Voir aussi Latour 2004. Pour une exégèse et une mise à jour du quadriparti de Heidegger, voir Harman 2010. "l'essence de la technique [...] qui n'est absolument rien de technique", menace de nous barrer l'accès à une rencontre plus essentielle avec ce qui est, la poiesis, ou la poétique révélant ce qui «fait paraître la chose présentée» (Heidegger 1958a: 37). À la place, notre confrontation agressive avec la nature transparaît même dans nos catégories philosophiques élémentaires. Des objets externes (Gegenstanden) sont supposés se dresser contre le sujet représentant et expérimentant, une relation établie par Descartes et approfondie par Kant (ibid. : 9-369 ). Dans arraisonnement, nous saisissons ce qui est comme une représentation interne, une image du monde (Weltbild) ou vision du monde (Weltanschauung); la relation entre les époques et les cultures est maintenant comprise comme «une confrontation de visions du monde 10" (ibid.: 35). Ainsi, l'ère de la technique est «l'époque des conceptions du monde" dans le sens où l'homme est maintenant l'être qui arraisonne et se représente le monde pour ses usages.

Une notion de ce que pourrait signifier la rencontre du monde autrement que comme représentation, interprétation ou matière première à exploiter apparaît dans les écrits poétiques de Heidegger sur la «chose". Dans son exemple, une cruche - une cruche précise - est ce qu'elle est non parce qu'elle a quatre côtés et un fond; une chose est plus qu'un collecteur de sensations, un objet physique, un gros morceau de matière formée ou un outil utile. Une cruche est plutôt un événement, un vide copieux qui reçoit le vin comme partie d'un rite collectif. "Ce qui fait de la cruche une cruche déploie son être dans le versement de ce qu'on offre." (Heidegger 1958c: 205) Comme le suggère l'étymologie selon laquelle "chose" (Ding) signifie originellement "rassemblement», la cruche, ou une autre entité modeste tel un pont, est un objet focal dont l'usage, pour les membres d'un groupe historique, concentre leurs modes communs d'être en relation entre eux et avec toutes les entités du monde - leur manière totale d'être-au-monde: «Dans le don de verser la terre et le ciel, les divinités et les mortels sont ensemble présents. " (Ibid. 11) En unissant ce quadriparti en une instance concrète, la "chose» fait surgir et dévoile un cosmos. La cruche est une chose cosmique.

Nous nous ouvrons à cet aspect des choses par la pensée «méditative", besinnlich, que Heidegger définit comme une "acquiescence" ou Gelassenheit, «sérénité». La pensée calculante, quant à elle, "ne s'arrête jamais, ne rentre pas en elle-même" (Heidegger 1966: 136): c'est la forme de pensée responsable des avions, radios, centrales électriques et bombes atomiques. Pour Heidegger, ces objets techniques, avec leur vitesse et leur annulation de la distance, tiennent l'être à distance; ils ne peuvent se comparer aux ponts ou cruches traditionnels et artisanaux qu'il considère comme des «choses" (ibid. : 145; 1958c: 206).

Mais de tels objets sont indéniablement des éléments constitutifs de l'être-au-monde contemporain; ils contribuent à la texture des pertinences qui forment notre «totalité équipementale». Ne sont-ils pas par conséquent les sites où notre clairière - le soubassement généralement négligé par lequel l'être se dévoile - devient visible? Devons-nous refuser aux objets technologiques leur propre ontopoétique démente? 


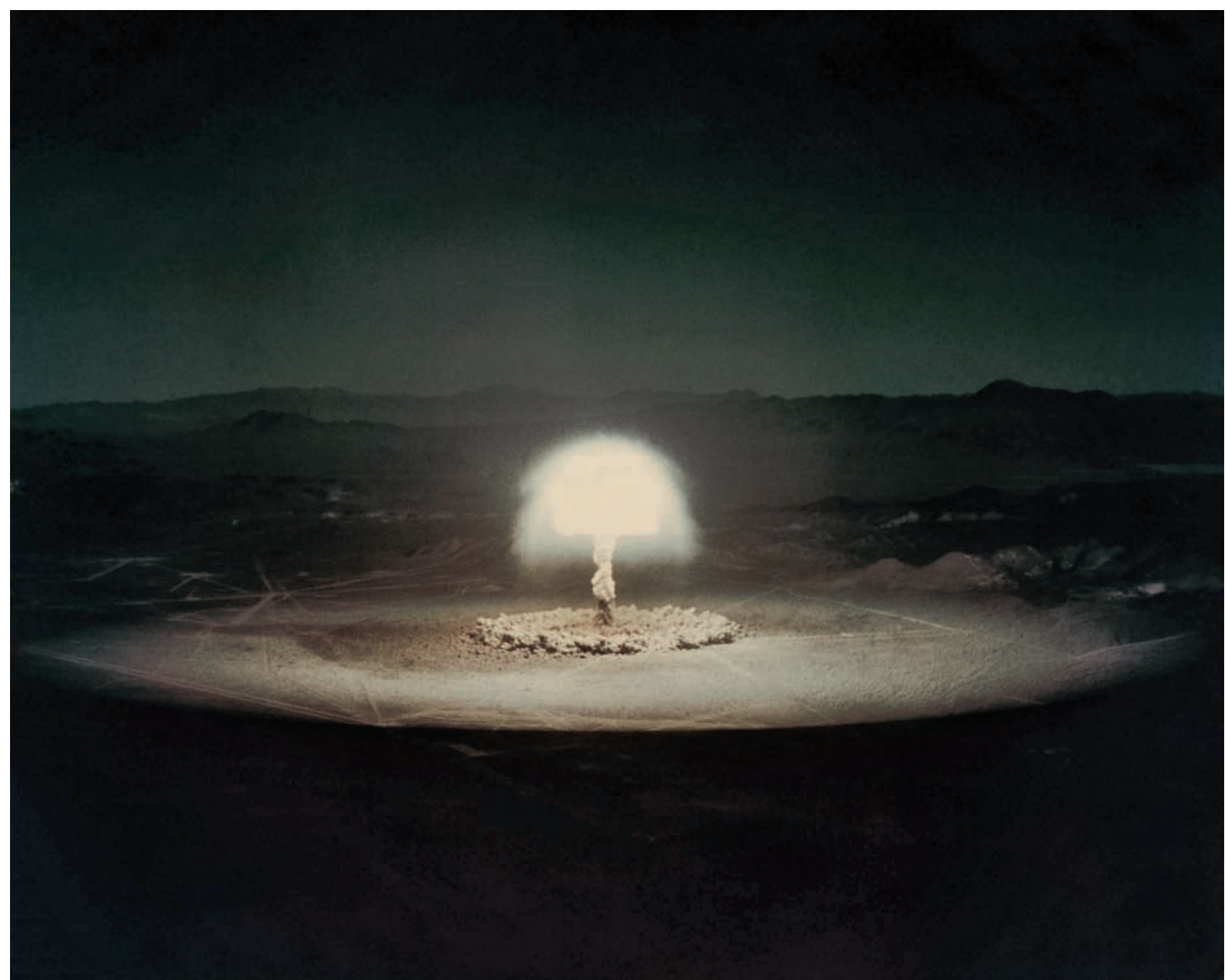

fig. 2

Essai atomique, s.d.

Photo Getty images. 


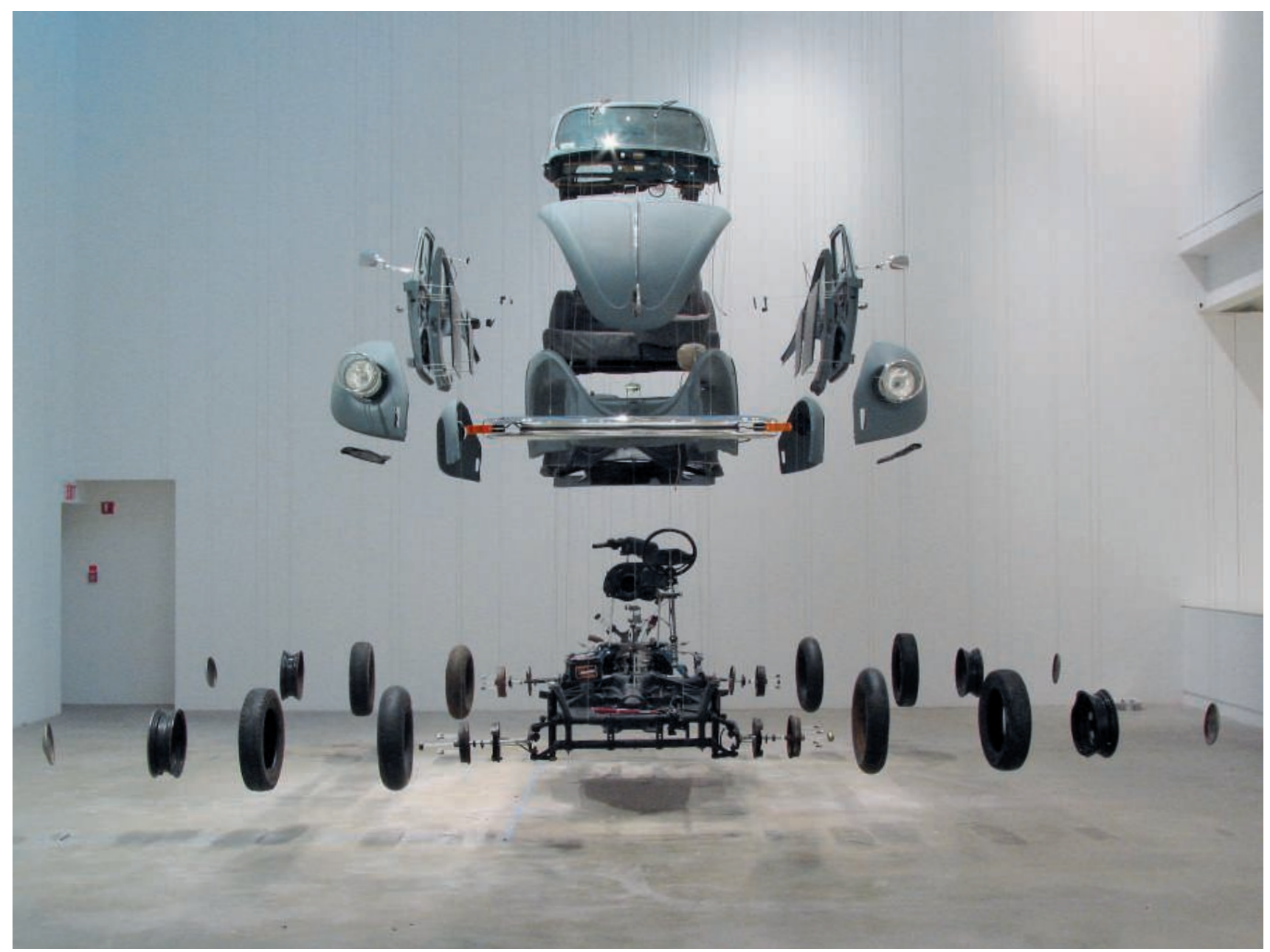

fig. 3

Damián Ortega, Cosmic

thing, divers matériaux

(acier, fer, Coccinelle

modèle 1983, plexiglas)

(c) Damián Ortega.

Courtesy White Cube and

Kurimanzutto, Mexico City. 


\section{Bug ${ }^{12}$, en suspens}

Jamais, dans l'apparition des choses, nous ne percevons d'abord et proprement [...] une pure affluence de sensations, par exemple de sons et de bruits [...]. C'est l'avion trimoteur qui fait ce bruit là-haut, et c'est la Mercedes que nous distinguons immédiatement d'une Adler. (Heidegger 1962a: 24)

Considérez la sculpture de la fig.3, éclatée comme un insecte disséqué à l'intérieur de l'Institut d'art contemporain de Philadelphie en 2002. L'artiste mexicain (et ancien propriétaire) Damián Ortega l'a intitulée Chose cosmique. Le titre d'Ortega est bien sûr ironique: que peut-on imaginer de moins «cosmique » que la carcasse morte d'une Coccinelle Volkswagen des années 1960, une machine encombrante probablement vouée à la casse sans ce détour (ou détournement) par le musée d'art? Une bagnole désarticulée et rien de plus. Et pourtant... dans le silence stérile de la galerie, l'objet semble être ce qu'il était et n'est plus: un outil fiable allant de soi. Sur les sièges usés, des taches de café renversé parlent du présent dilaté de la circulation les jours de semaine. Des bosses sur les pare-chocs suspendus gravent le somnambulisme de masse d'une ville surpeuplée. La chape des pneus porte la marque du trottoir et de la route de terre battue, d'arrêts abrupts et de courses inquiètes, et la possibilité ouverte de fuites vers l'ailleurs. Par la vitre, des paysages s'approchent et s'éloignent, océan et montagne, gratte-ciel et maison de brique; sur la peinture grise fanée est tracée l'action inlassable du soleil, de la poussière, de la pluie et de l'air, à laquelle s'ajoutent des restes de merde d'oiseau et de moustiques écrasés.

Loin d'un objet autonome (ou se mouvant seul, auto-mobile), le caractère compartimenté de la machine démondéisée est éclatant: tel un schéma de montage en trois dimensions, à l'échelle 1/1, l'installation fige travail, expertise, acier d'ingénierie de précision, verre, plastique et caoutchouc qui, mus par une force centripète, fusionnent dans ce rassemblement unique, de concert avec les choix importants de conception et les innovations matérielles qui la faisaient avancer - du moteur monté à l'arrière et refroidi à l'air et de la coque détachable à la suspension avec sa barre de torsion, alors novatrice. Elle réunit rues pavées, ateliers de mécanique, stationsservice et pompes à essence - qui, nous le savons bien, dépendent à leur tour de relations fragiles entre des individus puissants, des corporations insatiables, des gouvernements volatiles.

Ici convergent la lutte et l'apaisement entre pauvres et riches, entre travailleurs, profiteurs et consommateurs. Ces chemins remontent les histoires des nations jusqu'au fascisme industriel qui a ébranlé le berceau de Volkswagen: cette voiture fut d'abord appelée «KDF» (Kraft durch Freude, «la force par la joie») et la réplique de Hitler à Ford a puisé largement dans le holisme romantique pour offrir au consommateur individuel l'unité mystique et violente des nazis, faite de volonté, de sol et de Volk ${ }^{13}$. Sauvé par les Britanniques, Volkswagen a ouvert une usine à Puebla, au Mexique, en 1964 - transfert préparé de longue date par l'engagement allemand en Amérique latine remontant au moins aux voyages d'Alexandre de Humboldt et à son Cosmos. La dernière «vieille Coccinelle» - ou Vocho, comme on la surnomme au Mexique - a quitté la ligne de montage en 2003, mais l'usine
12. Bug signifie d'abord «insecte» en anglais (N.d.T.).

13. Voir Nelson 1970. Wolfsburg, le site de l'usine Volkswagen, fut conçu comme la cité idéale des travailleurs nazis. 
14. La trajectoire de la Volkswagen renvoie de façon inquiétante à celle des textes de Heidegger récupérés par l'existentialisme et la déconstruction pour leur potentiel critique et émancipateur. Pour une discussion nuancée sur les relations compliquées entre holisme romantique, "liberté " et totalitarisme, voir Harrington 1996.

15. Ou dans la version du chanteur pop Donovan d'inspiration zen:

"First there is a mountain then there is no mountain, then there is. "Voir Baas 2005. Sur les rencontres maladroites de Heidegger avec la philosophie asiatique voir les commentaires hilarants d'Hans Gumbrecht (2000). En art contemporain, Cosmic Thing a été rapproché de la mutilation sculpturale d'une DS Citroën (ou déesse) par le compatriote d'Ortega, Gabriel Orozco (Bush 2003: 129).

\section{ci-contre}

fig. 4

Baronne Elsa von FreytagLoringhoven et Morton Schamberg, God, 1917. Tirage gélatino-argentique, The Metropolitan Museum of Art, New York, Elisha Whittelsey Collection, Elisha Whittelsey Fund, 1973, 1973.637.

Photo (C) The Metropolitan Museum of Art, Dist. RMN-Grand Palais / image of the MMA. intégrée donne encore naissance à un millier de «nouvelles Coccinelle» par jour à destination du marché étatsunien.

Avec cette filiation, la métamorphose de la Coccinelle des années 1960 en une icône du néoromantisme - le véhicule préféré des disciples de Jack Kerouac et Neal Cassady revivant les errances de Meister Wilhelm sur l'Autobahn américaine - est une démonstration troublante de la flexibilité interprétative des objets techniques ${ }^{\mathbf{1 4}}$. Dans la galerie d'art américaine en 2002, le titre Chose cosmique rappelle ces associations contre-culturelles, avec une différence toutefois: à un moment de panne du dynamisme critique et créatif, les aspirations croissantes de l'art et de la politique des années 1960 semblent tristement dépassées, à reléguer au rang de curiosités muséologiques. La Coccinelle pré-anthropomorphisée d'Ortega apparaît désormais comme sa propre commémoration dans un rituel burlesque: la dépouille d'un ancêtre cher appelé à devenir un dieu oublié.

Cette douleur née d'un utopisme déçu nous alerte: peut-être avons-nous été naîfs en voulant à tout prix trouver du sens dans les salons de l'avant-garde calcinés par l'ironie? Si l'on voit seulement l'équanimité muette des pièces produites en masse et déconnectées, les fils de signification surdéterminés que nous venons de remonter se dénouent aussitôt; incapable d'être à la hauteur de son titre hyperbolique (Chose cosmique), l'objet redevient un outil cassé, parodiant la tendance au monumentalisme des institutions d'art. Mais peut-être est-ce ici, dans ce refoulement du sens, que nous nous approchons le plus d'une autre face du "cosmique", non pas comme plénitude, mais comme manque. L'immense objet, suspendu tel un système solaire ou un atome, offre un aperçu éphémère de l'«automobile" en tant qu'assemblage arbitraire, dépourvu d'essence - vide et finalement sans substance, comme tous les êtres qui naissent de la coproduction conditionnée (Komito 2001). L'objet flotte maintenant parmi les ondes de la philosophie orientale qui ont traversé l'art conceptuel occidental depuis les années 1960 au moins: d'abord il y a une Vocho, puis il n'y a pas de Vocho, puis il y en a une ${ }^{15}$.

L'objet technique peut être un simple outil. Cependant, un pont, une rame de métro, une machine à vapeur, un synthétiseur, un lave-linge, un fusil, une drosophile standard ou une souris génétiquement modifiée peuvent aussi révéler comment chacun de ces objets et le monde que les humains partagent avec eux accèdent à la présence. Comme les souliers du paysan de Van Gogh, la machine conservée et enchâssée d'Ortega, pourtant produite par l'industrie de masse et une ingénierie de précision, assure la locomotion quotidienne tout en rassemblant un cosmos.

\section{Des images du monde choséifiées}

À ce stade, le lecteur prudent pourrait s'inquiéter de la vitesse hasardeuse à laquelle nous empilons nos hypothèses. Comment être certain en effet que nos explorations, dans et hors de cette Volkswagen explosée, aient plus qu'un rapport éphémère avec les intentions de l'artiste ou avec le sens hic et nunc de l'exposition? De plus, une voiture n'est qu'une voiture - ou plutôt, la voiture conduite, négligée ou choyée est différente de la tâche d'un travailleur à la chaîne, de la migraine d'un mécanicien, de la messe en 


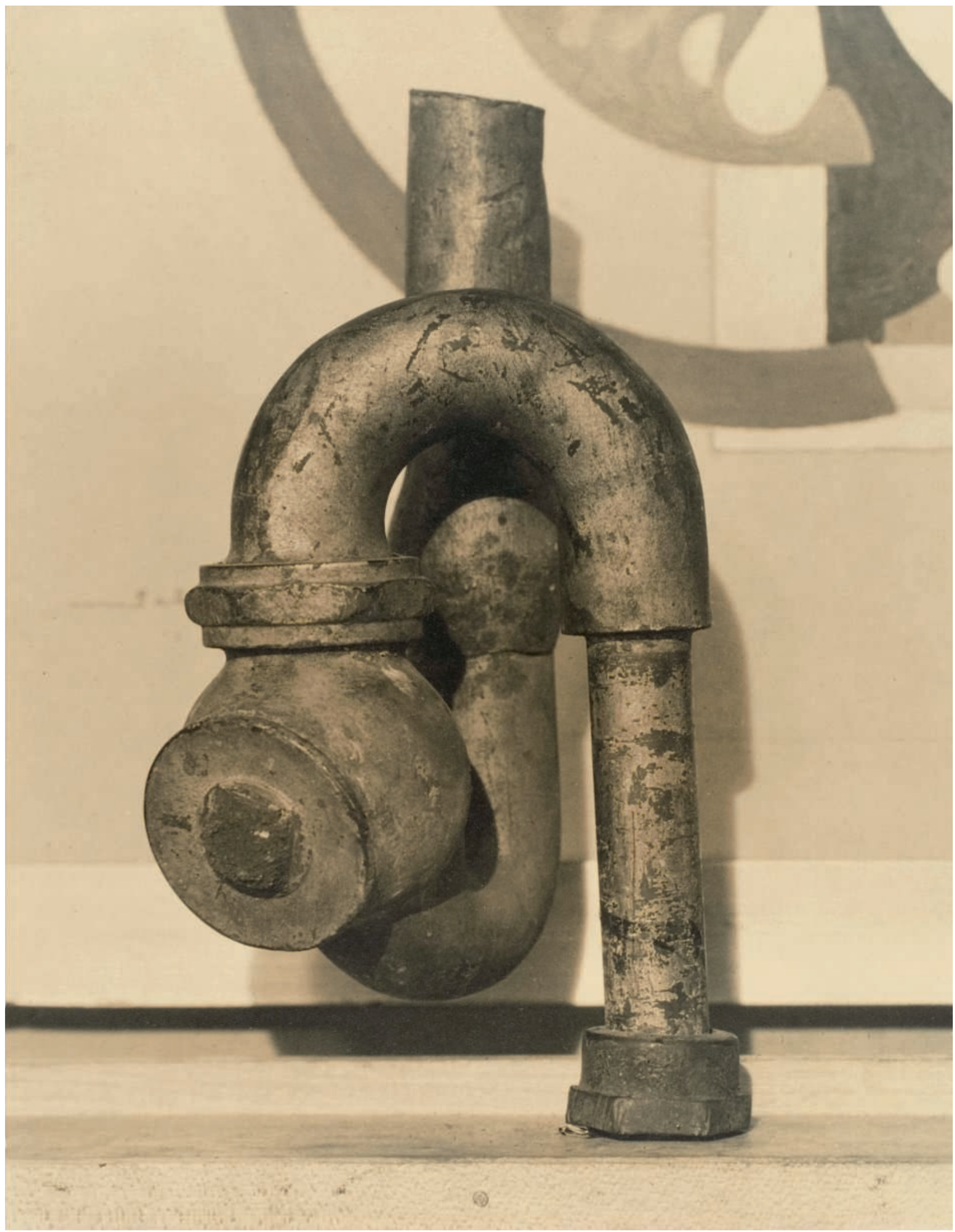


16. Pour un résumé péremptoire des critiques de la "culture", voir Abu-Lughod 1991; Bourdieu 1994; Marcus et Clifford (éd.) 1986 ; Wagner 1981.

Sur l'anthropologie postculturelle, voir Bunzl 2006.

17. Pour une palette d'acceptions de la notion de "cosmogramme" lors d'entretiens avec des historiens de l'art, des sciences et de l'architecture, des étudiants de Buckminster Fuller, des anthropologues, des astronomes et des survivants de la biosphère, voir Ohanian et Royoux (éd.) 2005 ; les contributions de Peter Sloterdijk, Patricia Falguières et Jean-Luc Nancy font particulièrement écho aux enjeux de cet essai. Geertz va dans le sens du cosmogramme avec sa remarque en note selon laquelle "les informateurs font souvent, voire habituellement, des interprétations de second degré - qui sont aujourd'hui appelées des "modèles indigènes" " (Geertz 1998: note 15) accéléré d'un physicien ou du jeu sérieux d'un artiste; seule la suspension artificielle du sens critique peut faire apparaître l'objet dans toutes ces dimensions à la fois. Et quelle que soit la prudence avec laquelle nous respectons les limitations de vitesse du discours académique, même le passager le plus tendu sera d'accord sur le fait que tout projet herméneutique est en proie à des doutes similaires: comment savoir si les significations que nous découvrons et les contextes que nous sollicitons dans nos interprétations d'un objet, texte ou événement sont pertinents pour leurs créateurs ou pour la gamme des utilisateurs et des publics contemporains?

De telles critiques hantent particulièrement l'anthropologie culturelle qui, pendant longtemps, a ancré ses interprétations dans la «culture», un concept très vilipendé dernièrement. Pour de nombreux chercheurs, postuler l'existence d'un système abstrait de significations, théories ou croyances qui sous-tend et détermine les conceptions et actions locales est un artefact de la relation asymétrique entre l'étranger-anthropologue «civilisé» et ceux qu'il étudie. De plus, la «culture» suppose l'existence d'un tout délimité, homogène et unifié, ce qui échoue à rendre compte des variations, nuances et exceptions ou de la palette d'attitudes que les acteurs peuvent activer selon les normes sociales et les traditions. La «culture» en tant que soubassement ou contexte explicatif s'appuie sur des hypothèses qui se sont révélées idéalistes, intentionnalistes, faussement homogénéisantes et anhistoriques ${ }^{16}$.

Plutôt que d'offrir une échappatoire au cercle herméneutique des interprètes de la culture, j'aimerais suggérer une solution modeste pour resserrer le cercle et en rendre les contours plus visibles, en partant du fait que les gens représentent réellement et publiquement l'univers comme un tout, pour eux-mêmes et pour les autres, sous forme d'objets - d'artefacts concrets, visibles. Même si nous suivons Heidegger et rejetons l'hypothèse selon laquelle tous les âges et toutes les cultures expérimentent le monde grâce à des représentations internes ou visions du monde, les groupes humains ont toujours créé des descriptions externes des éléments du cosmos et de leurs connexions, leurs propres explications sous forme d'étant discret de la totalité fugitive de l'être. Ce sont des cosmogrammes. Ces objets ont été construits pour rendre explicite la «chose cosmique ${ }^{\mathbf{1 7}}$ ». Les exemples sont légion: songeons à l'épique De la nature des choses de Lucrèce; aux mandalas tibétains à la fois plans de temple, cartes métaphysiques et aides à la méditation; aux mosquées qui sous leurs dômes unissent Dieu à la création sans le représenter directement; aux récits en images de la création et du salut et aux labyrinthes qui, dans les cathédrales, guident le pèlerin vers leur sens caché; aux rites dogons qui enveloppent d'une couverture un cube coiffé d'un cône pour jouer la fusion du ciel et de la terre; aux palais hindous plaçant le roi exactement au mitan du peuple, du gouvernement et des dieux; au Principia de Newton; à la réinvention high-tech du temple de Salomon dans la Nouvelle Atlantide de Bacon; aux encyclopédies, cartes et arbres de la connaissance de toute sorte; à l'implantation au sol des universités; aux architectures et aux moteurs d'information et de recherche; à l'Ulysse de Joyce; à l'Origine de Darwin; à Powers of Ten de Charles et Ray Eames; au Comment construire un univers qui ne s'effondre pas deux jours plus tard? de Philip K. Dick; à l'espace entre deux 
marches à Buenos Aires qui renferme, selon Jorge Luis Borges, «l'objet secret et conjonctural dont les hommes usurpent le nom mais qu'aucun homme n'a regardé - l'inconcevable univers ${ }^{18}$ ".

Ces choses exceptionnellement "denses " offrent un raccourci dans le cercle herméneutique. En un lieu et un temps donnés, de multiples cosmogrammes circulent dans un espace public et partagé. On obtient ainsi une idée de la "localisation cosmique» d'une entité, de sa place dans la chorégraphie des contextes culturels par triangulation avec de multiples cosmogrammes contemporains. Cela fournit une interprétation déterminée mais non définitive puisque l'entité se révélera différemment selon les cartes où elle est placée. De plus, concrets et publics, les cosmogrammes sont eux-mêmes continuellement exposés à des contestations, additions, suppressions et substitutions; une présentation de l'univers à valeur permanente ou universelle, que ce soit par Borges ou Rudolf Carnap, relève de la sciencefiction. De fait, l'acceptation de la partialité et de la faillibilité de tout cosmogramme - l'écart entre l'image d'unité qu'il renvoie et les entités réfractaires qu'il assemble - distingue cette approche des théories représentationnelles du savoir, de l'idéalisme de l'histoire traditionnelle des idées ou du déterminisme circonscrit et déterminé par des "visions du monde» internalisées, des "cosmologies» ou des "cultures». Dans la mesure où ils présentent une totalité tout en restant fermement dans le local et l'empirique, les cosmogrammes rendent possibles un holisme ouvert. Ils offrent des pistes d'unification sans exiger d'uniformité. On peut étudier comment les individus contestent ou transforment différents cosmogrammes et comment ils sélectionnent ou alternent entre les différents cadres temporels et référentiels que ceux-ci suggèrent ${ }^{19}$. Bien plus qu'une représentation, les cosmogrammes sont des propositions, des actes illocutoires et performatifs: ils démontrent comment faire des choses avec des mondes (Austin 1970).

Une approche historique de notre question initiale sur la relation entre la technologie moderne et le cosmos pourrait commencer, par exemple, par l'examen de cosmogrammes de la période où de nouvelles machines et systèmes techniques furent reconnus comme responsables de profonds bouleversements dans la vie quotidienne, quand la « révolution industrielle" a été identifiée comme telle pour la première fois. Dans cette perspective, j'ai étudié les représentations du cosmos de la première moitié du $x x^{e}$ siècle, surtout en France, en privilégiant la place qu'elles accordent aux nouvelles technologies: machine à vapeur, instruments électromagnétiques, daguerréotypes et autres techniques de communication de masse. En cette période de soulèvements incessants, un appel à une nouvelle doctrine susceptible d'unir la société provoqua une explosion de représentations du cosmos. Un grand nombre de ces schémas panoramiques, récits de création, slogans de ralliement et fragments cristallins ont atteint leur public sous forme de journaux, feuilletons et livres produits en masse par les presses à vapeur. D'autres cosmogrammes ont eu une forme non verbale: les tableaux et cartes des forces productives des ingénieurs du Conservatoire national des arts et métiers; le phalanstère de Charles Fourier; la salle construite à l'Observatoire de Paris pour l'astronomie populaire de François Arago; les Vues de la nature de Humboldt; les gigantesques temples de la femme conçus par les saint-simoniens;
18. Jorge Luis Borges, L'Aleph (1967: 209): "Je vis la mer populeuse, je vis l'aube et le soir, je vis les multitudes d'Amérique. Je vis dans une arrière-cour de la rue Soler le même dallage que j'ai vu il y a trente ans dans une maison de Fray Brentos; je vis des grappes, de la neige, du tabac, des veines de métal, de la vapeur ", une vision qui submerge le narrateur - "J'eus le vertige et je pleurai. » Selon les termes du rival du narrateur : "Quel observatoire formidable, mon cher Borges! » (Ibid. : 208 et 210)

19. Ainsi,

les cosmogrammes sont tout à fait utilisables dans l'« ethnographie du détail " prônée par Lila Abu-Lughod comme antidote aux généralisations induites par la "culture»

(Abu-Lughod 1991). Malgré ses visées généralisantes et universalisantes, tout cosmogramme a une localisation matérielle et une histoire propre et appartient à des pratiques et événements locaux irréductibles. Pour des comparaisons entre formes sociales dans lesquelles des cosmogrammes furent construits et discutés en Grèce et en Chine, voir Lloyd et Sivin 2005, notamment p. 174-187, et sur le concept même de cosmos élaboré par Héraclite et le Yi-King comme plan du cosmos, p. 269. 

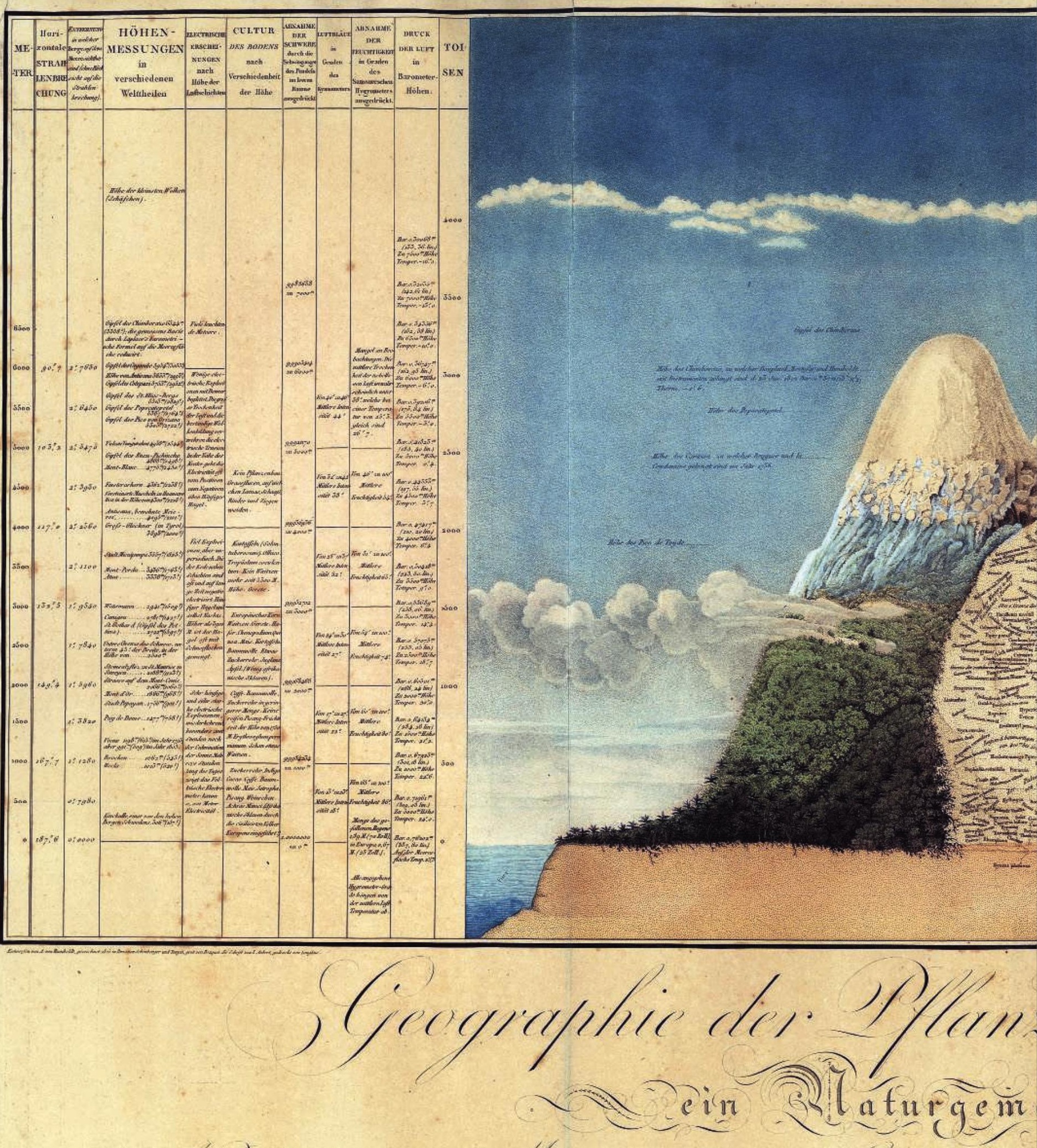

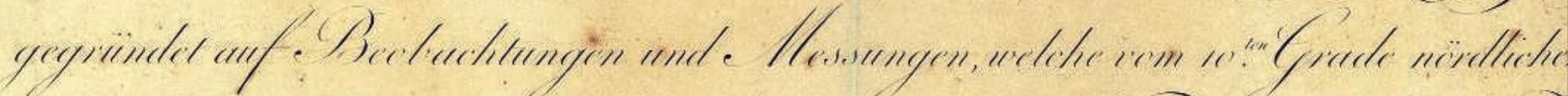

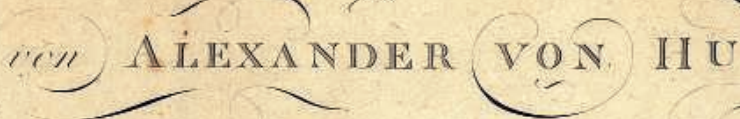


double page précédente

fig. 5

Friedrich Alexander Humboldt, Géographie des plantes dans les pays tropicaux. Étude des Andes, dessin de Schoenberger \& Turpin, imp. Langlois, Paris, 1803. Lithographie. HumboldtUniversitaet, Berlin, Germany / (C) HumboldtUniversitaet zu Berlin / Bridgeman Images. ou l'influente imago mundi de la religion de l'Humanité, le calendrier positiviste d'Auguste Comte.

Comme les œuvres tardives de Heidegger nous inciteraient à le penser, nombre de ces cosmogrammes du début de l'ère industrielle décrivaient ces nouvelles machines en tant qu'instruments destinés à encadrer, transformer ou exploiter la nature, avec la Terre (ou le système solaire, pour Fourier) comme réserve disponible de matière première censée satisfaire les besoins humains. Pourtant, là où Heidegger puisait souvent dans les thèmes romantiques pour s'opposer à la technologie moderne - comme quand il oppose le poème d'Hölderlin, Le Rhin, à la station hydroélectrique moderne installée sur le même fleuve -, nombreux furent les cosmologistes prétendant accéder à un rapport préréflexif, affectif, esthétique et holistique à la nature et à la source ineffable de l'être, tout en adhérant aux sciences nouvelles et à la technologie. Ils suggéraient des trajectoires alternatives vers la modernité, où les objets techniques et le savoir scientifique n'étaient pas perçus comme antithétiques de l'existence humaine «authentique» - la «proximité de l'être" ou l'« habiter poétiquement» de Heidegger; au début de l'ère industrielle, l'objectif et le subjectif, le mécanique et le romantique, le réaliste et le fantastique étaient intimement et étonnamment imbriqués. Ces fusions ont également eu lieu à travers des objets techniques qui canalisaient et convertissaient des fluides et des forces dynamiques souvent invisibles, ainsi que des cosmogrammes qui cherchaient à expliciter le nouvel ordre naturel et social incarné par ces «machines romantiques» (Tresh 2012).

Vers 1915, Rainer Maria Rilke écrivit ces vers repris plus tard par Heidegger: "L'esprit de notre temps emmagasine des forces en de vastes greniers, Lui qui est encore informe, tout comme cette énergie débordante qui lui vient de partout à la fois. Les temples, il les a oubliés." (Rilke 1991 [1923]: 51) Pour donner encore plus de poids à la vision inhabituelle de la technique du milieu du $x \mathrm{x}^{\mathrm{e}}$ siècle, pénétrons maintenant dans le bâtiment qui, telle une pépite rêvée dans le Paris de l'époque et édifiée de l'autre côté du globe, illustre la notion de cosmogramme et offre un contre-exemple à la complainte familière de Rilke et Heidegger.

\section{La technocratie rencontre la nécrocratie}

Dès ses premiers écrits comme apprenti polytechnicien auprès de Saint-Simon dans les années 1820, Auguste Comte insista sur la nécessité de remplacer l'autorité perdue de l'Église par un nouveau «pouvoir spirituel» pour en finir avec l'anarchie de la Révolution. L'humanité n'était plus unie par la foi en des dieux imaginaires et des idoles; à son stade final, elle croirait seulement en ce qu'on peut voir et prouver. Afin d'unifier les sciences, d'offrir une description cohérente du monde et de la place de l'humanité en son sein, et de guider le progrès de l'industrie, la science elle-même deviendrait une religion: les philosophes formés à la science en seraient les nouveaux prêtres. Comte, qui s'effondra mentalement et physiquement dans les années 1840, recouvra la santé grâce à son «interlocutrice séraphique", Clotilde de Vaux. II comprit alors qu'il avait négligé les émotions et les contributions des femmes à l'humanité. Son nouveau «système positif » était un mode de vie complet, modelé sur l'Église catholique; le fétichisme, première religion, serait restauré, mais l'amour pour des objets externes serait vidé de tout 
mysticisme. Un siècle avant la déclaration de Heidegger selon laquelle «seul un dieu peut nous sauver», Comte nomma une divinité post-théologique devant laquelle il s'agenouilla: I'Humanité, le Grand Être, auquel nous et tous ceux qui ont vécu appartiennent 20 (Comte 1890). Le Catéchisme positiviste et sa «table de sociolâtrie» énonçaient le dogme et les rites de la religion de l'Humanité, avec ses prières, exercices spirituels, hiérarchie cléricale, et descriptions des bannières et oriflammes verts (couleur de l'espoir) qui décoreraient ses temples. Comte mourut avant que ses projets ne se réalisent. Mais, en 1895, le verbe positiviste prit corps au Brésil.

Le Templo da Humanidade s'élève dans le quartier de Gloria, dans une rue poussiéreuse nommée Benjamin-Constant, non pas en hommage au libéral romantique suisse mais au positiviste qui rédigea la Constitution de la République brésilienne. La cour envahie par les arbres, les pierres et la grille rouillée trahissent leur âge, et en 2009 le toit s'est lamentablement effondré. Mais les colonnes et la façade, inspirées du Panthéon à Paris, sont encore debout ${ }^{21}$. Cette église en béton, verre, marbre et bois poli incarne le système de Comte. La cour elle-même est un espace sacré et pédagogique. Au tympan se trouve un compas dont une branche est toujours tournée vers Paris, pays natal de Comte et Mecque de la nouvelle religion universelle. Les marches copient l'échelle des sciences du cours de philosophie positive, dans l'ordre où celles-ci ont atteint la positivité, du «sol» des Mathématiques vers la Sociologie et, sur le palier, la Moralité, instituée et insufflée dans l'Église. Lors d'une visite en 2006, au faîte du temple flottait le drapeau de l'Humanité - globe blanc sur fond vert - flanqué de ceux du Brésil et de la France. En gros caractères, la devise inchangée s'étale au fronton: «L'amour pour principe et l'ordre pour base, le progrès pour but » (fig. 6).

L'intérieur vaste est baigné d'une délicate lumière d'aquarium, traversant les rideaux et réfléchie sur les murs dans des verts menthe et chartreuse, tons d'un autre siècle. Le long des murs, sous les drapeaux des nations du monde, des plaques affichent les étapes fondamentales de la connaissance et de la société: Espace, Logique, Terre, et chacune des sciences du cours de philosophie positive ainsi que Fétichisme, Polythéisme, Monothéisme et Métaphysique. Sur les murs se détachent les bustes, peints en couleurs primaires, de grands bienfaiteurs de la civilisation, qui ont aussi donné leurs noms aux mois du calendrier positiviste: nous passons sous le regard de Moïse («Première Théocratie») et d'Homère («Épopée ancienne»), jusqu’à Frédéric II («Politique moderne») et Bichat («Science moderne»). Soulignant l'importance de l'activité et de l'invention dans le système de Comte, ainsi que son orientation profondément technique, le premier buste que nous voyons à gauche est celui de Gutenberg, sous le titre «Industrie moderne».

Comme la sculpture ornant la façade, ces statues donnent à l'intérieur une impression funèbre, vaguement sépulcrale - comme si Diderot et d'Alembert avaient collaboré avec Madame Tussaud. Au-dessus de la sortie est inscrit le slogan justifiant ce qu'on pourrait appeler la nécrocratie de Comte: "Les vivants sont nécessairement toujours et de plus en plus gouvernés par les morts. "Selon Comte, nous devons tout, science, art, institutions et langage, à ceux qui ne sont plus. Le temple est musée et
20. Pour des travaux récents sur la philosophie et la religion de Comte, voir Grange 1996; Petit (éd.) 2003; Karsenti 2006; et, en anglais, les travaux de Mary Pickering.

21. Ce monument majeur de l'histoire philosophique et politique, négligé par le gouvernement brésilien, cherche à se reconstruire en centre de recherche international; voir le site https://blogdaipb. wordpress.com 


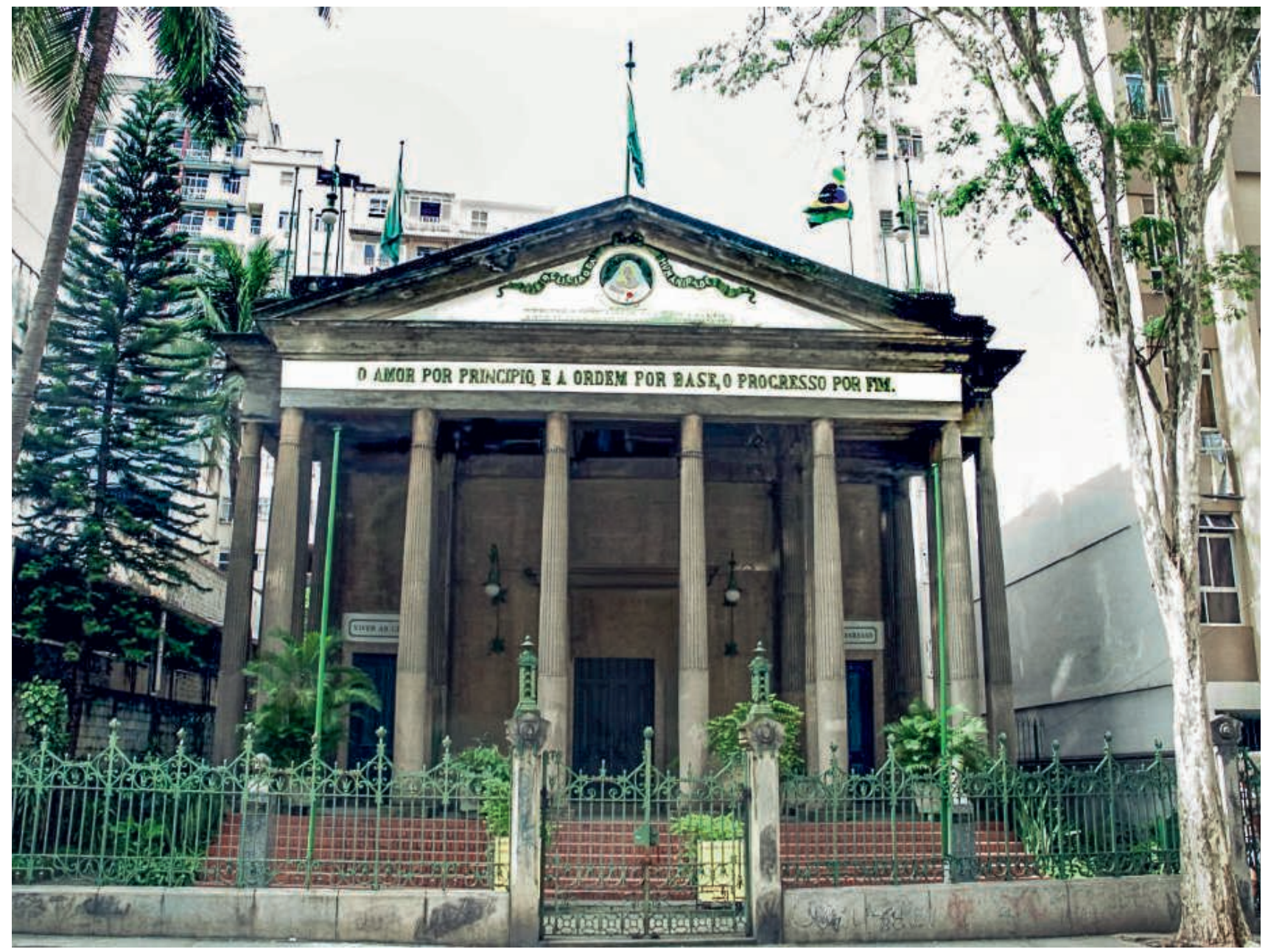

fig. 6

Façade du Templo da Humanidade, Rio de Janeiro, 2006. (C) Photo Margarete Vöhringer. mausolée à la fois, un lieu où apprendre, et se souvenir en même temps commémorer - , les fondateurs et les principes fondateurs de notre monde. Outre ces reliques des saints positivistes, les tableaux de Comte et de Clotilde de Vaux occupent l'espace autour de l'autel: elle, pleurant en «veuve éternelle»; elle, étendue sur son lit de mort, Auguste agenouillé à son chevet; elle, présence éthérée, murmurant à l'oreille du grand prêtre tandis qu'il écrit.

Au centre se trouve un podium d'où les prêtres vont rallier et lier, tel est le rôle de la religion (et l'étymologie du mot). Le successeur en sociologie de Comte, Émile Durkheim, a révélé dans Les Formes élémentaires de la vie religieuse que, par-delà les histoires d'origine et les divinités des religions, l'objet réel du culte est la société elle-même et les sentiments qu'elle engendre et renforce (Durkheim 1960 [1912]). Le temple de l'Humanité rend cette logique explicite. La déesse ici objet de culte est $A$ Humanidade, titre du portrait suspendu derrière la chaire, où une femme avec le visage de Clotilde de Vaux s'élève vers les cieux, vêtue d'une robe vaporeuse, tenant contre son sein un bambin: la race humaine, passée, présente et future.

En tant que cosmogramme, le temple n'est ni simplement un symbole ou une représentation, ni une réflexion ou une projection; c'est un instrument, une machine destinée à fonder, maintenir et étendre un ordre naturel et social spécifique ainsi que les émotions qui le soutiendront. Fondé par Miguel 
Lemos qui, comme les autres leaders positivistes, était étroitement lié à l'École polytechnique du Brésil, il a servi de point de ralliement à une génération de réformateurs en politique et en éducation qui ont franchi ses murs alors qu'ils reconstruisaient l'État brésilien. Des positivistes tels que Teixeira Mendes et Benjamin Constant ont été une force majeure d'opposition à la fois à l'Église et à l'Empire, et de fondation de l'État moderne; ils ont dirigé la lutte contre l'esclavage, proclamé la république, rédigé la Constitution et initié la réforme agricole et le développement des chemins de fer et de l'industrie.

Un rouleau effiloché, caché près de l'autel, se défait pour révéler, sur du papier millimétré du $\mathrm{XIX}^{\mathrm{e}}$ siècle, une ébauche peinte en vert, bleu et jaune par le peintre positiviste Décio Vilares: la première esquisse du drapeau brésilien. Sa devise, Ordem e Progresso, fait écho à celle de l'entrée de l'église et traverse un cercle bleu figurant le firmament comme on le voyait depuis Rio le soir où la république a été proclamée, chacune des étoiles évoquant l'un des États du Brésil. C'est l'union, en deux dimensions, de la terre, du ciel, des mortels et de la divinité de l'Humanité présentée suivant le style de communication de l'internationalisme du tournant du siècle - et sous les couleurs portées plus récemment par Pelé, Ronaldo et leurs millions de supporters (Garcia-Parpet 2002).

Dans cet édifice, les relations entre les humains et tous les autres êtres sont exposées et réaffirmées. Le temple abrite une clairière dans laquelle un peuple vivra, préservant son histoire et projetant son futur. II réunit un cosmos, telle une œuvre d'art ou une chose heideggerienne. Mais en son sein, dans le monde qu'il encercle, un espace est maintenu ouvert - assez bizarrement, un espace sacré - pour les sciences théoriques et quantitatives, pour les œuvres et les machines de l'industrie et de la technologie modernes.

\section{Laisser advenir les choses techniques}

Nous pourrions aisément décrire le positivisme de Comte comme la confirmation des diagnostics les plus sombres de Heidegger sur la science moderne et la technique. Pour Comte, en effet, tout savoir est relatif aux besoins humains: les sciences encadrent la nature afin d'agir sur elle. Quant à l'«impérialisme planétaire», le titre du Système de l'industrie positive. Traité sur l'action totale de l'humanité sur la planète parle de luimême. Cependant, de nombreux éléments de son système - comme ceux de ses contemporains mécaniquement romantiques - ne riment pas avec l'arraisonnement technique, le détachement, l'uniformité ou l'exploitation effrénée. Par son insistance sur les méthodes, les normes épistémologiques et les concepts propres à chaque science, l'épistémologie de Comte ressemble au «réalisme pluriel » qu'Hubert Dreyfus trouve chez Heidegger ou, tout à fait différemment, à ce que l'anthropologue brésilien Eduardo Viveiros de Castro a appelé, suivant les dispositions cosmologiques des peuples de l'Amazonie, le multinaturalisme. La définition de l'«espace», dans la Synthèse subjective, comme le «Grand Milieu », absence fondatrice à la base de tous les ordres sociaux et intellectuels, renvoie à l'insistance de Heidegger sur la mystérieuse absence de fondement absolu du Dasein. Avec sa définition de la terre comme le «Grand Fétiche» - qui résonne 
22. Comte 1900, que Grange voit comme une proto-écologie (Grange 1996); Dreyfus 1991: 262, sur le "réalisme pluriel" de Heidegger; Viveiros de Castro 2005

23. Sur des formes alternatives de modernité, voir Mitchell (éd.) 2000 Pour les cosmologies dans I'histoire des sciences, voir Tresch 2014.

24. On pourrait comparer le «laisserêtre " de Gelassenheit avec le "pas de recul» que Schiller recommande comme la première étape vers l'«état esthétique "

dans Lettres sur l'éducation esthétique de l'homme, premier ouvrage à avoir déploré «le désenchantement du monde ": "L'homme doit donc, afin

d'échanger la passivité contre l'autonomie, une détermination passive contre une détermination active, être momentanément libre de toute détermination et traverser un état de simple déterminabilité. II doit en conséquence revenir d'une certaine manière à l'état négatif de simple indétermination dans lequel il se trouvait avant qu'une chose quelconque eut fait une impression sur ses sens " (Schiller 1943 [1795], Vingtième Lettre: 168). étrangement avec les cultes du candomblé foisonnant dans le quartier de Gloria à Rio -, le système de Comte enjoint la gratitude, l'altruisme, et par-dessus tout l'amour pour la planète et ses habitants. À la fois inventeur du mot "altruisme» et l'un des premiers philosophes à aborder des problématiques écologistes à l'époque industrielle, Comte propose des modes d'entente entre les humains et la terre qui détonnent avec l'abord agressif de l'arraisonnement ${ }^{22}$.

L'antidote le plus simple à la conception totalisante et fataliste de Heidegger de la modernité technique reste cependant le choix du pluriel pour "cosmogrammes". Le temple de l'Humanité de Rio est l'un des innombrables objets, à l'efficacité variable, qui ont eu pour but d'unifier et de banaliser les mondes dévoilés par l'industrie et la technique. II existe deux exemples contrastés: à Brasilia, la capitale qui a remplacé Rio, des rues rectilignes et des bâtiments administratifs incurvés dessinés par l'élève du Corbusier, Oscar Niemeyer, offrent une vision lisse et géométrique d'un monde transparent, quantifiable, et en fin de compte difficile à vivre (Holston 1989). Le long de la côte, à Porto Alegre, le Forum social mondial s'est réuni en 2005 pour ébaucher des modes de coordination globale destinés à contrecarrer la destruction accomplie par les «seigneurs de la terre » d'aujourd'hui et à mettre en œuvre le slogan visionnaire «Un autre monde est possible ${ }^{\mathbf{2 3}}$ ».

Dans Gelassenheit, Heidegger admet de façon cryptique que l'«ouverture au secret» et le fait de laisser advenir les choses «nous rendent possible de séjourner parmi les choses d'une manière toute nouvelle"; ce qui promet "une autre terre, un autre sol, sur lequel tout en restant dans le monde technique [...] nous puissions nous tenir et subsister ${ }^{24}$ » (Heidegger 1966: 146). Un pas vers cette autre façon de vivre - pas que Heidegger n'a jamais franchi - peut consister à laisser la technologie être davantage qu'une chose quelconque, et à admettre qu'elle n'est pas forcément porteuse d'un univers unique qui serait partout le même, hostile à la pluralité et au dynamisme de la vie et de l'être. La démarche consistant à aborder les objets techniques comme des choses cosmiques et à suivre les contours des images du monde tracées par les cosmogrammes - industriels et autres - va dans ce sens. Sur ce chemin, pour ceux d'entre nous qui étudient la science et la technologie, la tâche est à partager avec les anthropologues: recueillir et faire connaître des usages et des objets qui rendent des mondes visibles, compatibles ou opposés, en défendant la clairière qu'ils gardent ouverte à ce qui est, a été et pourrait être.

\section{ci-contre}


" $O$ day and night, but this is wondrous strange"

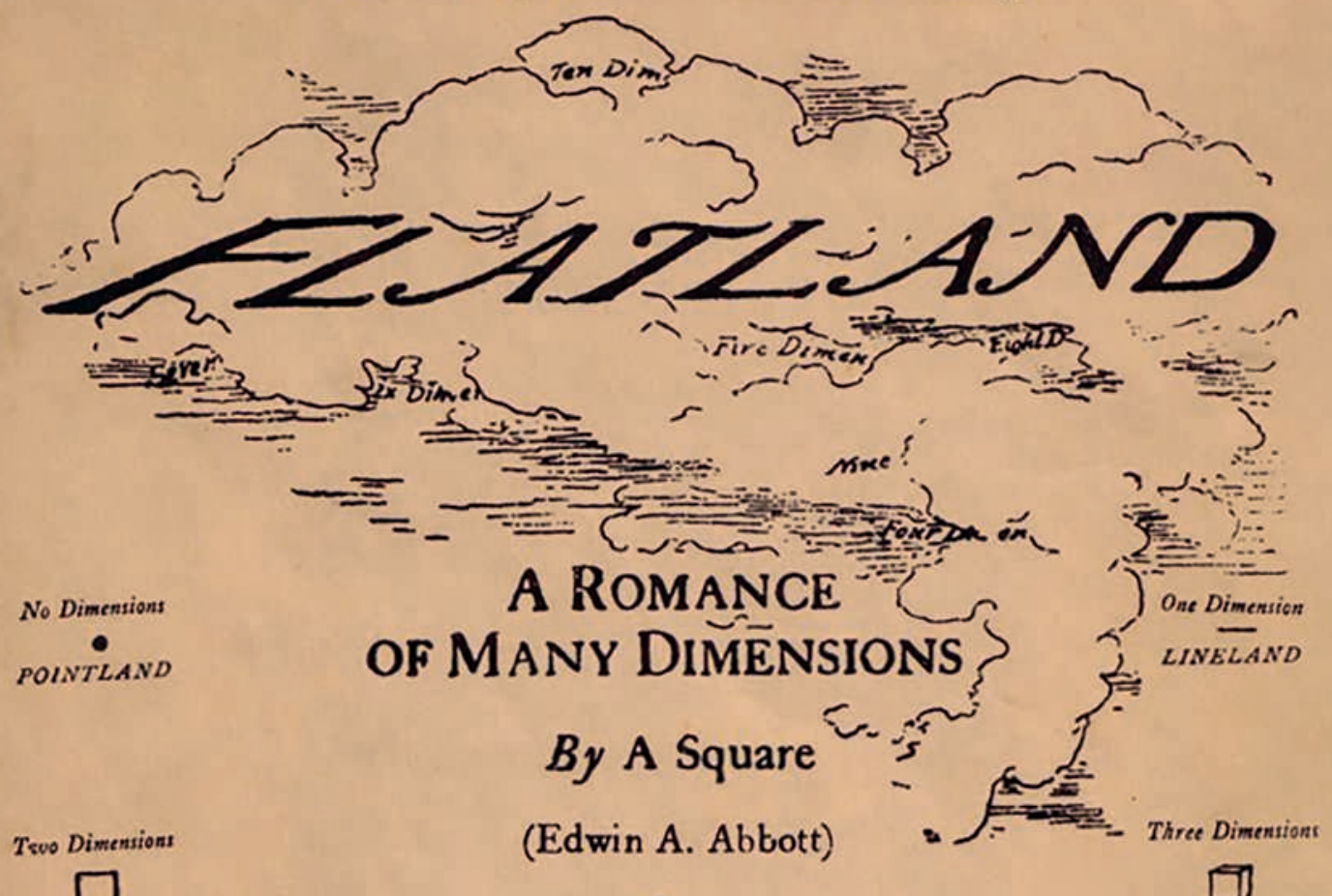

$\square_{\text {FLATLAND }}$

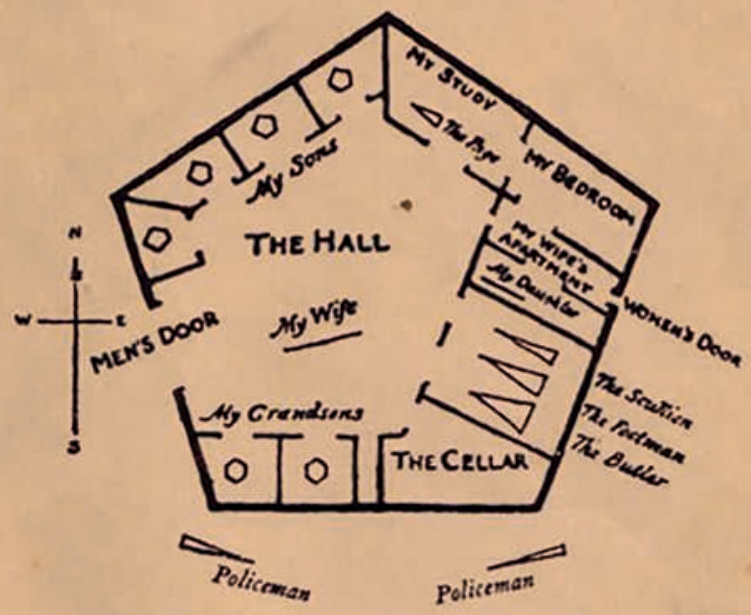

SPACELAND

"And therefore as a stranger give it welcome."

\section{BASIL BLACKWELL - OXFORD}

Price Seven Shillings and Sixpence net 


\section{Abu-Lughod, Lila}

1991 "Writing against culture», in Richard G. Fox (éd.), Recapturing Anthropology: Working in the Present. Santa Fe, School of American Research Press: 137-162.

\section{Austin, John}

1970 Quand dire c'est faire. Paris, Seuil.

\section{Baas, Jacquelynn}

2005 Smile of the Buddha: Eastern Philosophy and Western Art from Monet to Today. Berkeley, University of California Press.

\section{Borges, Jorge Luis}

1967 L'Aleph. Paris, Gallimard («L'imaginaire »), trad. René L.-F. Durand.

\section{Bourdieu, Pierre}

1994 Raisons pratiques. Sur la théorie de l'action. Paris, Seuil.

\section{Bunzl, Matti}

2006 «Were we wrong? Rethinking the postmodern paradigm", communication au Colloquium for the history of the human sciences, Philadelphie, University of Pennsylvania.

\section{Bush, Kate}

2003 "First Take", Artforum 41.

\section{Cardwell, Donald S.L.}

1972 Turning Points in Western Technology: A Study of Technology, Science and History. New York Science History Publications.

\section{Chakrabarty, Dipesh}

2000 Provincializing Europe: Postcolonial Thought and Difference. Princeton, Princeton University Press.

\section{Comte, Auguste}

1890 Catéchisme positiviste ou Sommaire exposition de la religion universelle en treize entretiens entre une femme et un prêtre de l'Humanité, $3^{e}$ éd. Paris, Larousse.

1900 La Synthèse subjective d'Auguste Comte, $2^{e}$ éd. Paris, Fonds Typographique.

\section{Daston, Lorraine}

2004 "Speechless ", in Lorraine Daston (éd.), Things That Talk: Object Lessons from Art and Science. New York, Zone: 9-26.

\section{Deleuze, Gilles}

1986 Foucault. Paris, Les Éditions de Minuit.

\section{Dijksterhuis, Eduard Jan}

1961 The Mechanization of the World-Picture. Oxford Oxford University Press.

\section{Dreyfus, Hubert}

1991 Being-in-the-World: A Commentary on Heidegger's Being and Time, division I. Cambridge, MIT Press.

1992 «Heidegger's history of the being of equipment ", in Dreyfus et Hall (éd.) 1992: 173-185.

\section{Dreyfus, Hubert et Hall, Harrison (éd.)}

1992 Heidegger: A Critical Reader. Oxford, Blackwell.

\section{Durkheim, Émile}

1960 [1912] Les Formes élémentaires de la vie religieuse. Paris, PUF.

\section{Farias, Victor}

1987 Heidegger et le nazisme. Paris, Verdier.

\section{Garcia-Parpet, Marie-France}

2002 "Les usages de la pensée française dans le Brésil du XIX ${ }^{\ominus}$ siècle: la question raciale, Auguste Comte et Arthur de Gobineau ", in Michel Bourdeau et François Chazel (éd.), Auguste Comte et l'idée de science de l'homme. Paris, L'Harmattan: 221-237.

\section{Geertz, Clifford}

1998 "La description dense", Enquête [En ligne], 6 :

http://enquete.revues.org/1443.

\section{Gordon, Peter}

2010 Continental Divide: Heidegger, Cassirer, Davos. Cambridge, Harvard University Press.

\section{Grange, Juliette}

1996 La Philosophie d'Auguste Comte: science, politique, religion. Paris, PUF.

\section{Gumbrecht, Hans}

2000 "Martin Heidegger and his Japanese interlocutors: About a limit in Western metaphysics ", Diacritics 30: 83-101.

\section{Habermas, Jürgen}

1992 «Work and Weltanschauung: The Heidegger controversy from a German perspective", in Dreyfus et Hall (éd.) 1992: 186-208.

\section{Harman, Graham}

2002 Tool-Being: Heidegger and the Metaphysics of Objects. Chicago, Open Court.

2010 L'Objet quadruple. Paris, PUF.

\section{Harrington, Anne}

1996 Reenchanted Science: Holism in German Culture from Wilhelm II to Hitler. Princeton, Princeton University Press.

\section{Heidegger, Martin}

1958a La Question de la technique, in Essais et conférences. Paris, Gallimard («Tel»), trad. André Préau $9-48$

1958b Bâtir Habiter Penser, in ibid. : 170-193.

1958c La Chose, in ibid. : 194-223.

1962a Origine de l'œuvre d'art, in Chemins qui ne mènent nulle part. Paris, Gallimard, trad. Wolfgang Brokmeier: 13-98.

1962b L'Époque des conceptions du monde, in ibid. : 99-146.

1966 "Sérénité ", Questions III et IV Paris, Gallimard («Tel»), trad. André Préau: 133-182

1971 Qu'est-ce qu'une chose? Paris, Gallimard («Tel»), trad Jean Reboul et Jacques Taminiaux.

1986 Etre et Temps. Paris, Gallimard ("Bibliothèque de philosophie»), trad. François Vézin.

\section{Holston, James}

1989 The Modernist City: An Anthropological critique of Brasília. Chicago, University of Chicago Press.

\section{Hughes, Thomas}

2004 Human-Built World: How to Think about Technology and Culture. Chicago, University of Chicago Press.

\section{Ihde, Don}

1979 Technics and Praxis. Dordrecht, Reidel.

1990 Technology and the Lifeworld. Bloomington, Indiana University Press.

2004 "Has the philosophy of technology arrived? A stateof-the-art review », Philosophy of Science 71 : 117-131.

\section{Karsenti, Bruno}

2006 Politique de l'esprit: Auguste Comte et la naissance de la science sociale. Paris, Hermann. 


\section{Kittler, Friedrich}

1992 Discourse Networks 1800-1900. Palo Alto, Stanford University Press.

\section{Komito, David}

2001 Nagarjuna: Psychologie bouddhiste de la vacuité.

Schoten, Kunchab.

\section{Latour, Bruno}

2004 «Why has critique run out of steam? From matters of fact to matters of concern ", Critical Inquiry 30: 225-248.

\section{Latour, Bruno et Weibel, Peter (éd.)}

2005 Making Things Public: Atmospheres of Democracy. Karlsruhe et Cambridge, ZKM-MIT Press.

\section{Lloyd, Geoffrey et Sivin, Nathan}

2005 The Way and the Word: Science and Medicine in Early China and Greece. New Haven, Yale University Press.

\section{Marcus, George et Clifford, James (éd.)}

1986 Writing Culture: The Poetics and Politics of Ethnography. Berkeley, University of California Press.

\section{Marx, Leo}

2000 The Machine in the Garden: Technology and the Pastoral Ideal. Oxford, Oxford University Press.

\section{Mitchell, Timothy}

1988 Colonising Egypt. New York Cambridge University Press.

\section{Mitchell, Timothy (éd.)}

2000 Questions of Modernity. Minneapolis, University of Minnesota Press.

\section{Nelson, Walter}

1970 Small Wonder: The Amazing Story of the Volkswagen Beetle. Boston, Little, Brown

\section{Noble, David}

1999 The Religion of Technology: The Divinity of Man and the Spirit of Invention. New York, Penguin.

\section{Ohanian, Melik et Royoux,} Jean-Christophe (éd.)

2005 Cosmograms. New York, Lukas \& Sternberg.

\section{Petit, Annie (éd.)}

2003 Auguste Comte: trajectoires positivistes (1798-1998). Paris, L'Harmattan.

\section{Rilke, Rainer Maria}

1991 [1923] "Septième élégie ", in Élégies de Duino. Paris, Actes Sud («Babel»), trad. Maximine.

\section{Rorty, Richard}

1993 «Wittgenstein, Heidegger and the reification of language", in Charles Guignon (éd.), The Cambridge Companion to Heidegger. Cambridge, Cambridge University Press: 337-357.

\section{Rouse, Joseph}

1987 Knowledge and Power: Toward a Political Philosophy of Science. Ithaca, Cornell University Press.

\section{Schaffer, Simon}

1989 «Glass works: Newton's prisms and the uses of experiment * in David Gooding, Trevor Pinch et Simon Schaffer (éd.), The Uses of Experiment. Studies in Natural Sciences. Cambridge, Cambridge University Press.

\section{Schiller, Friedrich}

1943 [1795] Lettres sur l'éducation

esthétique de l'homme, texte original et traduction française Robert Leroux [En ligne]; http://classiques.uqac.ca

\section{Sloterdijk, Peter}

1998-2004 Sphären I-III. Francfort, Suhrkamp.

\section{Tresch, John}

2012 The Romantic Machine: Utopian Science and Technology after Napoleon. Chicago, University of Chicago Press.

2014 "Cosmologies materialized: History of science and history of ideas ", in Darrin M. McMahon et Samuel Moyn (éd.), Rethinking Modern European Intellectual History. New York, Oxford University Press: 153-177.

\section{Viveiros de Castro, Eduardo}

2005 «From multi-culturalism to multi-naturalism " in Ohanian et Royoux (éd.) 2005: 137-156.

2012 "Cosmologies:

Perspectivism ", Hau Master Class Series I.

\section{Wagner, Roy}

1981 The Invention of Culture. Chicago, University of Chicago Press.

page 24 et ci-contre Baronne Elsa von FreytagLoringhoven et Morton Schamberg, God, 1917. Tirage gélatino-argentique, The Metropolitan Museum of Art, New York, Elisha Whittelsey Collection, Elisha Whittelsey Fund, 1973, 1973.637.

Photo (C) The Metropolitan Museum of Art, Dist. RMN-Grand Palais / image of the MMA.

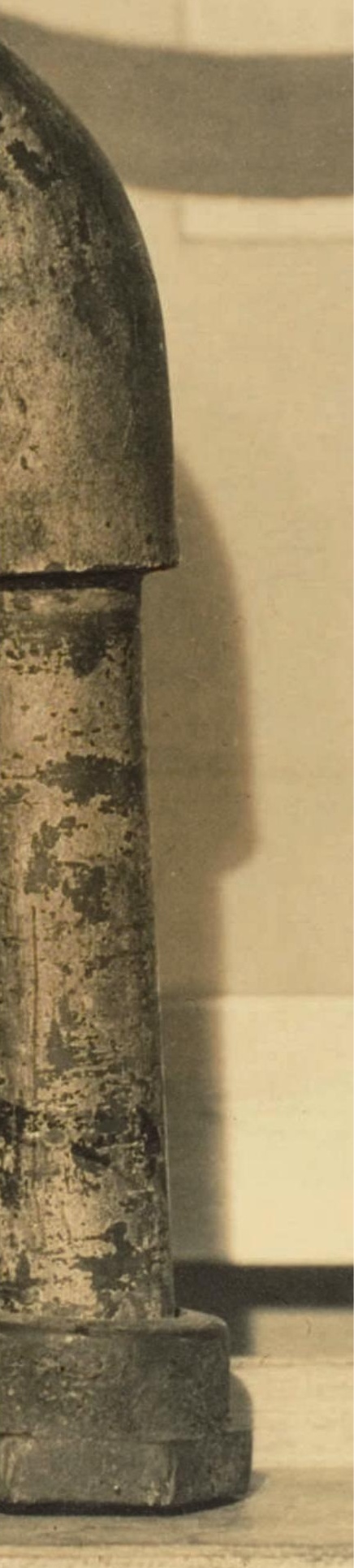

